\title{
P300 Evoked Potential and Risk of Mild Cognitive Impairment Progression to Alzheimer' s Dementia: A Literature Review
}

Marco Túlio Gualberto Cintra ${ }^{*}$, Moacir Cesar Silva Tavares², Sérgio Augusto Gomes², Thiago de Oliveira Gonçalves², Luciana Cristina Matos da Cunha ${ }^{3}$, Denise Utsch Gonçalves ${ }^{4}$, Edgar Nunes de Moraes ${ }^{1}$ and Maria Aparecida Camargos Bicalho'

1 Jenny de Andrade Faria Institute for the Elderly of the Clinical Hospital, Federal University of Minas Gerais, Brazil

${ }^{2}$ Medical student of the Federal University of Minas Gerais, Brazil

${ }^{3}$ Speech therapist, Federal Universty of Minas Gerais, Brazil

${ }^{4}$ Otorhinolaryngology Department of the Federal Universty of Minas Gerais, Brazil

\begin{abstract}
Introduction: Great efforts have been made to develop diagnostic methods for precisely screen patients with mild cognitive impairment $(\mathrm{MCl}$ ) who will progress to dementia. Many biomarkers have been studied for that. However, their high costs, small availability and need for invasive procedures sometimes make these biomarkers of limited usefulness. With the advantages of its low cost and being non-invasive, the P300 evoked potential (EP) is one methods under investigation.

Objective: To assess whether the P300 evoked potential can estimate the risk of $\mathrm{MCl}$ progression to Alzheimer's dementia (AD).

Methods: Review of the PubMed database using the descriptors: "evoked potentials", "Alzheimer's disease" and "mild cognitive impairment".

Results: We selected eight among 929 articles after applying the exclusion criteria. From the articles we concluded that the electrode placed at the parietal region is the most effective and that the latency increase and amplitude decrease of the electrode reading are related to the higher risk of progression from $\mathrm{MCl}$ to a diagnosis of AD. Most of the selected studies sustain P300 EP to estimate the progression risk from $\mathrm{MCl}$ to AD. However, the low number of studies, small sample size and heterogeneous results are pointed as important limitations.
\end{abstract}

Conclusion: The P300 represents a promising method to estimate the likelihood of the MCI progression to AD. However, more studies are needed to support P300 for daily clinical practice.

Keywords: Evoked potentials; P300; mild cognitive impairment; Alzheimer's disease

\section{Introduction}

Mild cognitive impairment (MCI) is a recent concept and refers to patients who have a cognitive decline not enough to fill the diagnostic criteria's for dementia [1,2]. It is divided into amnestic and nonamnestic sub-groups. MCI carriers exhibit an annual rate of dementia progression about 12 percent whereas elderly with preserved cognition have a rate of 1-2 percent of progression to a diagnosis of dementia. The risk is higher in the amnestic subtype [3-5].

There has been an increasing interest in studying subjects with MCI, as a model for the development of early diagnostic tests considering the upcoming disease-modifying drugs, which could benefit patients in the initial or prodromic phase of $\mathrm{AD}$ [6]. According to the National Institute on Ageing and the Alzheimer's Association the biomarkers based on cerebrospinal fluid (CSF) analysis and functional imaging methods are highlighted among the more recently incorporated diagnostics tests for MCI [7].

However, the use of these methods is not universally available and they can add costs to the diagnosis of MCI and AD. Additionally, searching for CSF markers requires lumbar puncture, an invasive procedure, even more technically defiant to be performed in the elderly [8]. For such reasons developing non-invasive, low-cost and reproducible diagnostic methods is now a necessity, what rose up the interest in evoked potentials (EP) recorded by electroencephalography (i.e. event-related potentials), particularly the component P300, which is sensitive enough to monitor the brain electrical activity, has neither cultural nor educational influence has low cost and is non-invasive [9].

$\mathrm{EP}$ is brain electrical responses to somatosensorial stimuli, mainly visual and auditory stimuli, which should be preferably repetitive at randomized intervals for being processed latter by computers. EP has amplitudes ranging from a few hundredths to $5 \mu \mathrm{V}$ microvolt and requires a precise electrode positioning and the processing of multiple stimuli to extract them from the electroencephalographic recordings (background activity) [10].

The exam should be conducted in an electrical and acoustic isolated environment and the patient must be seated. The most commonly used technique for obtaining P300 is through acquisition of auditory evoked potential. Capturing responses from low-resistance electrodes (less than $5 \mathrm{~K} \Omega$ ) positioned at the frontal, vertex and parietal midline in relation to the bi-auricular reference using the auditory oddball paradigm. 300 stimuli are delivered every 1 second, being 20 percent at $2000 \mathrm{~Hz}$ (stimuli rarely identified by the patient) and 80 percent at 750-1000 Hz [11].

After the stimulus, the evoked potentials can be captured with a delay of 80 to $700 \mathrm{~ms}$ [12]. These evoked potentials are classified in

*Corresponding author: Marco Túlio Gualberto Cintra, 90/404 Katia Teodoro Aguiar Street, Belo Horizonte- Minas Gerais, Brazil, 30730-250, Tel: 55-31-36466108; E-mail: marcotuliocintra@gmail.com

Received September 07, 2015; Accepted October 27, 2015; Published Octobe 31, 2015

Citation: Cintra MTG, Tavares MCS, Gomes SA, Gonçalves TdeO, Cunha LCMda, et al. (2015) P300 Evoked Potential and Risk of Mild Cognitive Impairment Progression to Alzheimer's Dementia: A Literature Review. J Neurol Neurophysiol 6: 322. doi:10.4172/2155-9562.1000322

Copyright: (c) 2015 Cintra MTG, et al. This is an open-access article distributed under the terms of the Creative Commons Attribution License, which permits unrestricted use, distribution, and reproduction in any medium, provided the original author and source are credited. 
Citation: Cintra MTG, Tavares MCS, Gomes SA, Gonçalves TdeO, Cunha LCMda, et al. (2015) P300 Evoked Potential and Risk of Mild Cognitive Impairment Progression to Alzheimer's Dementia: A Literature Review. J Neurol Neurophysiol 6: 322. doi:10.4172/2155-9562.1000322

Page 2 of 5

exogenous potentials (N100, P200, N200) because they are strongly influenced by the physical characteristics of the stimulus (intensity, frequency and others) and in endogenous potentials (P300) which only reflects cognitive skills [13]. The component P300 is defined as the highest positive peak occurring between 250 and 500 milliseconds after a rare (occasional relevant-'target') stimuli, occurring after the two negatives components $\mathrm{N} 100$ and N200 and the positive component P200 [11,14].

The auditory P300 is an evoked potential of long latency, which allows the study of the aural cortex in the central nervous system. It represents the cortical activity comprising the discriminative, the integrative and the attention skills, being an excellent indicator of the cortical processing speed. The P300 raised a strong curiosity in the research community since it is frequently abnormal in cognitive impaired patients [15].

Amplitude and latency (time elapsed between onset of stimulus and emergence of the peak) are calculated for the peak [11]. The component P300 is influenced by the information processing, such as attention, stimulus evaluation, judgment, and decision-making [16]. One can subdivide the component P300 into two sub-components, P3a and P3b, reflecting different activities as stated by Squires et al (1975) with the former being more related to passive attention and the latter being particularly related to active attention and memory [17].

The early components $\mathrm{N} 100$ and P200 waves reflect the sensorial processing and are of low value for the diagnoses or monitoring cognitive impairment in AD. Otherwise, the N200 and P300, which reflect the cognitive processing, can be useful for monitoring the cognitive impairment. The source of N200 is the cortex frontal responsible for the conscious discrimination, evaluation and selection of stimuli. [12,18] The P300 is generated in many area of the cortex, mainly in the temporoparietal cortex, and reflects cognitive processes as attention, recognizing and classification of stimulus, and also work memory and decision making. Therefore, it is supposed that P300 is more useful than
N200 in the diagnoses and monitoring cognitive deficit [18].

The component P300 has an increased latency in AD patients compared to those with preserved cognition, especially in the analysis of the parietal electrode responses [18]. However, there is no consensus on the value of $\mathrm{P} 300$ for diagnosis of MCI and on its application to determine the risk of MCI progression to $\mathrm{AD}$ [19].

Our objective with the present study is to assess whether the P300 evoked potential can contribute to estimate the risk of MCI progression to $\mathrm{AD}$.

\section{Methods}

We conducted a search in the PubMed database in June 2015 using the MeSH descriptors: "evoked potentials", "Alzheimer disease" and "mild cognitive impairment". We first selected meta-analysis and systematic review studies, founding ten articles, with two meta-analyses, one of which dealing with the P300 evoked potential. Starting from these meta-analyses we used the instrument "see all" until saturation of new scientific studies regarding the objective of the present study. We ended curbing the selection of articles to systematic reviews, metaanalyses, cohort and case-control studies, written in English, Spanish or Portuguese. Cross-sectional studies, case reports, articles published in other languages were excluded, also as articles using EP but not the P300 component and articles exclusively reporting MCI progression to non-Alzheimer dementia. The article selection flowchart for this review is depicted in Figure 1.

\section{Results}

A total of 929 studies were initially found, resulting in 16 articles for full reading after evaluation of the abstracts. Of these, we selected eight articles who investigated the role of P300 evoked potential for assessing the risk of MCI progression to $\mathrm{AD}$, seven being observational studies and one meta-analysis. Table 1 describes the sample and the methodological features of the seven observational studies.

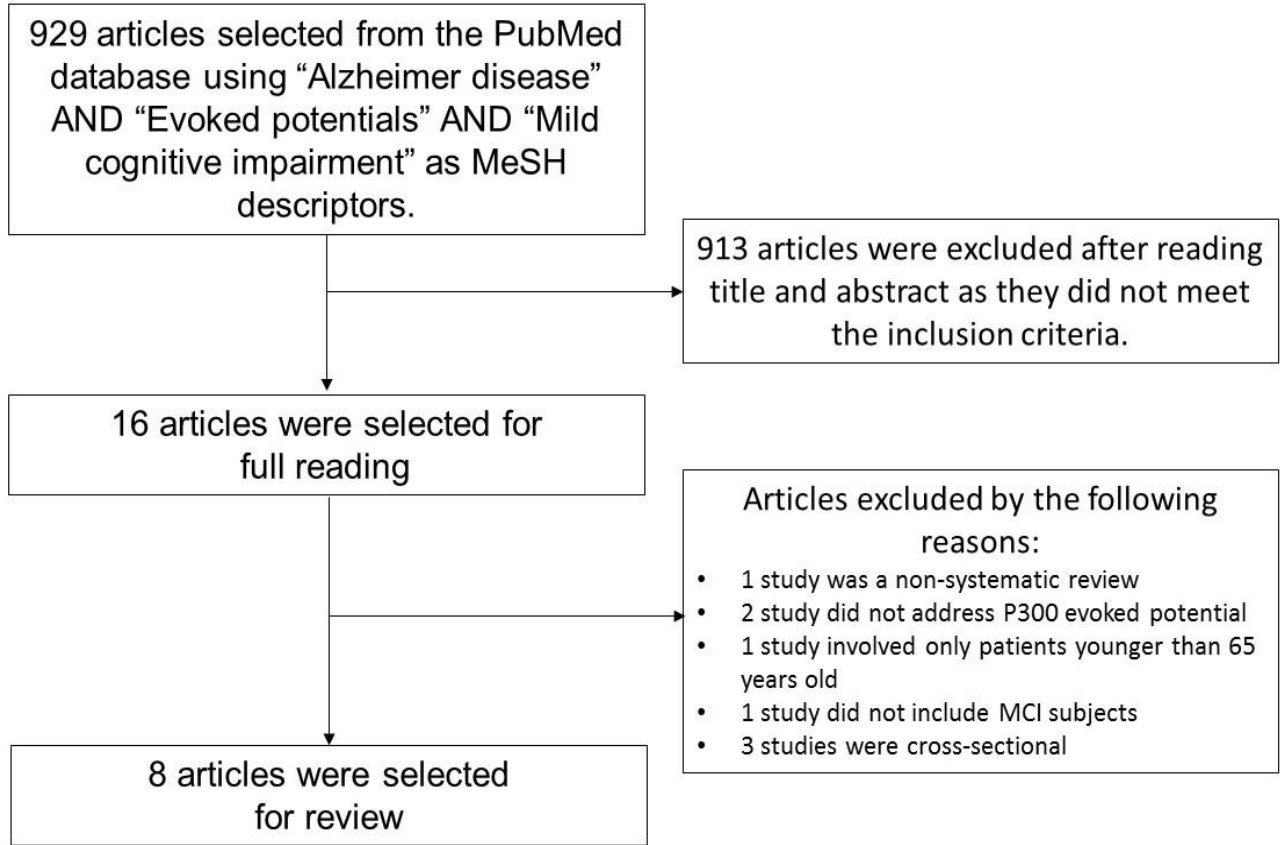

Figure 1: Flowchart of article selection for the literature review (Mild cognitive impairment $-\mathrm{MCl}$ ) 


\begin{tabular}{|c|c|c|c|c|c|}
\hline & Sample & Type of Study & Follow-up period & Diagnostic criteria for $\mathrm{MCl}$ & Diagnostic criteria for $A D$ \\
\hline Gironell A, et al 2005 & 116 controls & Nested case-control and cohort & $27.7 \pm 4 \mathrm{mo}$ & Petersen's criteria & $\begin{array}{l}\text { DSM-IV and NINCDS- } \\
\text { ADRDA }\end{array}$ \\
\hline Golob EJ, et al 2007 & $\begin{array}{c}44 \text { elderly controls, } 22 \\
\text { adult controls, } 41 \mathrm{MCl} \text { and } \\
14 \mathrm{AD}\end{array}$ & Prospective cohort & $6 \mathrm{yrs}$ & Petersen's criteria & NINCDS-ADRDA \\
\hline $\begin{array}{l}\text { Papaliagkas V, et al } \\
2008\end{array}$ & $91 \mathrm{MCl}$ and 30 controls & Prospective cohort & $23 \pm 3 \mathrm{mo}$ & Petersen's criteria & DSM-IV \\
\hline Lai CL, et al 2010 & $\begin{array}{c}20 \mathrm{AD}, 18 \mathrm{MCl} \text { and } 14 \\
\text { controls }\end{array}$ & Prospective cohort & $12 \mathrm{mo}$ & Petersen's criteria & $\begin{array}{l}\text { DSM-IV and NINCDS- } \\
\text { ADRDA }\end{array}$ \\
\hline $\begin{array}{l}\text { Papaliagkas VT, et al } \\
2010\end{array}$ & $20 \mathrm{MCl}$ & Prospective cohort & $11 \pm 5.9 \mathrm{mo}$ & Petersen's criteria & DSM-IV \\
\hline Bennys K, et al 2011 & $71 \mathrm{MCl}$ and 31 controls & Prospective cohort & $12 \mathrm{mo}$ & Petersen's criteria & uninformed \\
\hline $\begin{array}{l}\text { Papaliagkas VT, et al } \\
2011\end{array}$ & $22 \mathrm{MCl}$ and 30 controls & Prospective cohort & $14 \pm 5.2 \mathrm{mo}$ & Petersen's criteria & DSM-IV-TR \\
\hline
\end{tabular}

Alzheimer's dementia: AD; Mild cognitive impairment: MCI; Month: mo; Diagnostic and Statistical Manual of Mental Disorders: DSM; NINCDS-ADRDA: National Institute of Neurological and Communicative Disorders and Stroke and the Alzheimer's disease and Related Disorders Association

Table 1: Methodological aspects of the selected studies.

Gironell A et al. performed a nested case-control study in which 116 patients with subjective memory complaints but normal cognition were recruited. These individuals were followed up for a mean period of $27.7 \pm 4$ months and their cognition was evaluated for outcome. After this period, 30 patients had normal cognition, 30 progressed to MCI, 28 progressed to $\mathrm{AD}$, and six progressed to other dementias. The follow-up of 22 patients were not completed. The authors compared the EP results obtained at the beginning and at the end of the 12 and 24 months of follow-up. [16]. There was a significant difference between the groups at baseline and after 24 months. However, the P300 latency values were similar in controls and in MCI patients, but they were increased in patients that evolved to AD. Therefore, despite an increase in the latency is associated with a 3.75 greater risk for developing $\mathrm{AD}$, there is no latency patterns related to a MCI diagnoses. These results suggest that MCI patients with higher latency have a greater risk to evolve to $\mathrm{AD}[16]$.

The limitations of the study are related to the fact that only results from the electrode placed on the parietal region were assessed and the P300 amplitude was not evaluated. In addition, part of the AD patients were taking anticholinesterase drugs, what could increase the risk of false-positive results [16].

Lai CL et al conducted a prospective cohort study involving 20 patients with mild AD (Clinical Dementia Rating Scale=1) who were taking donepezil, 18 patients with MCI and 14 controls who were followed up for 1 year. At the end of the study, the authors demonstrated that the MCI group exhibited changes in the P300 latency using vertex and parietal electrodes [20]. In both electrodes, the authors observed an increased latency after the follow up period only in AD group. Therefore, it is possible that the increased latency observed at the vertex and parietal electrodes could be associated with progression from MCI to $\mathrm{AD}[20]$.

The cognitive abilities screening instrument (CASI) was employed for the cognitive diagnosis. However, there was a possible selection bias in the formation of the study groups, since the questionnaire had not been validated at the time of the study. Other limitations are the small sample size and the short follow-up period to investigate cognitive changes [20].

Papaliagkas V et al. followed up $91 \mathrm{MCI}$ patients and 30 controls for 14 months, with five elderly patients converting to AD. Part of the sample had age below 65 years old. P300 evoked potentials were assessed by using parietal and vertex electrodes. The study reports that neuropsychological tests were performed during the follow-up of the patients. Nevertheless, only the mini-mental state exam (MMSE) was cited. After the 14-month follow-up period, it was found that MCI patients exhibited significant increase in latency and decrease in the amplitude threshold $(\mathrm{p}=0.05)$. However, patients with MCI who converted to $\mathrm{AD}$ had no increase in P300 latency and amplitude. These results suggest that the changes in P300 latency and amplitude could not be associated with progression from MCI to $\mathrm{AD}$ [21].

Papaliagkas VT et al carried out a prospective cohort study with 53 MCI patients who had been submitted to P300 evoked potential and dosage of the level of CSF $\beta$ - amyloid (1-42) obtained by lumbar puncture. The same electrode positioning cited in the study by Papaliagkas VT et al (2008) was evaluated. Of all the patients, only 20 were prospectively followed up during a period of $11 \pm 5.9$ months, with five progressing to $\mathrm{AD}$ and the others remaining cognitively stable. As a result, it was observed that a higher latency of P300 was associated with a higher risk of AD progression [22].

Another study by Papaliagkas VT et al is an analysis of a subgroup of subjects included in an earlier work (Papaliagkas VT et al) which was followed up for $23 \pm 3$ months, comprising $22 \mathrm{MCI}$ patients and 30 controls [21,22]. Three MCI patients progressed to AD. The same electrode positioning was evaluated as in the earlier studies [21,22]. During the follow-up period, it was observed a significant increase in latency and a non-statistically significant decrease in the amplitude of the P300 potential. The MCI patients who progressed to AD exhibited no significant change in latency and amplitude compared to those who did not [23].

It is noteworthy that the above-cited study by Papaliagkas VT et al is an analysis of a subgroup of patients who had been followed up by the same authors in Papaliagkas VT et al [21,22]. The same occurs with the other study by Papaliagkas VT et al regarding the 2008 publication $[21,23]$. It is also observed that the sample sizes were very small in two studies $[21,22]$ and the follow-up periods too short, the only exception being the 2011 study, which adds up to $23 \pm 3$ months of follow-up [21-23].

Bennys $\mathrm{K}$ et al following up for an year a prospective cohort, comprising $71 \mathrm{MCI}$ patients and 31 controls without any cognitive complains, observed a worsening of the symptoms in 41 patients (58\%) of the MCI group, although not corresponding to a progression to dementia. Among these 41 patients, 17 had a progression to AD. Comparing the P300 results of patients, which had the MCI progression with those that remained stable, they could demonstrate 
that the increased $\mathrm{P} 3 \mathrm{~b}$ latency in the parietal region was related to $\mathrm{AD}$ conversion [24].

In Bennys K et al study, the small sample size as well as the formation of even smaller subgroups, reducing the power of the findings despite the significant $\mathrm{P}$ values are important limitations. In addition, the criteria for defining $\mathrm{AD}$ progression was objectively described such as only a 3-point decline in the MMSE [24].

Golob et al prospectively followed up, for 6 years, 134 patients: 66 controls (22 younger than 65 years), 41 with MCI (28 with singledomain amnestic MCI and 13 with multiple-domain amnestic MCI) and 14 with $\mathrm{AD}$. Among the $41 \mathrm{MCI}$ patients, 15 converted to $\mathrm{AD}$ at an annual rate of $10 \%$, mainly from the multiple-domain amnestic MCI subgroup. The P300 evoked potential was assessed by using three electrodes (frontal, vertex and parietal). The MCI patients who converted to $\mathrm{AD}$ had intermediate latency compared to the groups with $\mathrm{AD}$ patients and those with stable MCI. However, no statistically significant difference in latency was found between the group of $\mathrm{MCI}$ patients who converted and the group with stable MCI [25].

In this study, approximately $50 \%$ of the MCI patients were on anticholinesterase drugs, what could have affected the results of P300. Nevertheless, statistical adjustments were made to compensate this. The small sample of MCI patients is another limitation of the study [25].

In a Meta-Analysis, conducted by Jiang $\mathrm{S}$ et al., 13 studies were selected among 367 . In the selected studies, only four had a prospectively approach while the others were done using a crosssectional design 15 . With regard to the cross-sectional studies it was observed that the P 300 latency had a significant P-value when comparing the normal cognition, MCI and AD groups, while the P300 amplitude was significant only when the normal cognition and MCI groups were compared. It was also observed that only the parietal electrode was significant for the amplitude, otherwise the parietal and vertex electrodes were statistically significant for the latency. They equally observed that the auditory stimulus was superior to visual stimulus for P300 acquisition, which can be initially explained by the fact that auditory stimuli are more studied and less heterogeneous than the visual results [19].

The four prospective studies selected in the meta-analysis had an initial sample of 135 patients with MCI, decreasing to 124 after one year of follow-up. The works by Golob EJ et al, Papaliagkas V et al and Papaliagkas V et al. $[21,23,25]$ were not included in this meta-analysis. The results showed that the decreased amplitude and the increased latency of P300 could anticipate not only the progression of MCI (not necessarily to dementia), but also confirm a more severe clinical presentation. Nevertheless, the authors of the meta-analysis detected a trend bias for publishing only studies with positive findings [19].

\section{Discussion}

The selected studies showed that the electrode placed on the parietal region produced the best results for assessment of the MCI progression. The results obtained with this electrode pointed to an increased latency and decreased amplitude of P300 as a predictive evidence of disease progression. However, most of the studies did not necessarily evaluate the progression of MCI to AD but only the cognitive worsening, even when it was not progressing to $\mathrm{AD}$. Therefore, it remains obscure whether changes in both the amplitude and the latency of P300 are sensitive enough to be used for detection of MCI conversion to $\mathrm{AD}$ $[16,19-25]$.
The small sample sizes and the relatively short follow-up period of most of the selected studies jeopardize the power of the announced results [20, 22-24]. The high heterogeneity found among the studies comprised in the meta-analysis conducted by Jiang $S$ et al (2015) could endanger its results. They also pointed to a trend for publishing studies only with positive results [19]. It is not possible either to know the sample size of the eight articles selected in our study, since the three studies published by Papaliagkas VT et al had used the samples [21-23].

Another interesting aspect is that a significant part of the studies demonstrated both an increase in the latency and a decrease in the amplitude of P300 in MCI subjects during the follow-up period $[16,19,20,23,25]$. However, the results are discordant regarding the role of P300 evoked potential in discriminating between stable MCI patients and those with MCI converting to AD. Bennys $\mathrm{K}$ et al., Papaliagkas VT et al and Gironell et al reported that changes in the P300 evoked potential can be used to estimate which MCI patients are more likely to progress to $\mathrm{AD}[16,22,24]$. Nevertheless, in the cohort studies conducted by Golob EJ et al, Papaliagkas V et al and Papaliagkas VT et al, no changes in amplitude and latency of P300 were observed $[21,23,25]$.

The limitations of the present study rise from the fact that only the PubMed database was searched and studies published in languages other than English, Spanish and Portuguese were also excluded, which can determine the absence of relevant scientific articles related to the theme.

The selected studies support the P300 evoked potential can be used to predict the risk of MCI patients to progress AD. The described limitations are frequently associated to methodological problems and do not exclude the possible efficacy of P300 [9]. Therefore, future studies can confirm its utility to define prognosis in MCI patients.

We have concluded that the P300 evoke potential, which is noninvasive, low-cost and insensitive to cultural aspects, is a promising method for predicting the chance of $\mathrm{MCI}$ progression to $\mathrm{AD}$. Nevertheless, there is still a lack of evidence for the routine use of this method, limiting it to research use only. Therefore, more studies are needed to define the application of P300 as a diagnostic method for determination of the MCI progress to $\mathrm{AD}$.

\section{References}

1. Petersen RC, Smith GE, Waring SC, Ivnik RJ, Tangalos EG, et al. (1999) Mild cognitive impairment: clinical characterization and outcome. Arch Neurol 56: 303-308.

2. Petersen RC (2004) Mild cognitive impairment as a diagnostic entity. J Intern Med 256: 183-194.

3. Petersen RC, Negash S (2008) Mild cognitive impairment: an overview. CNS Spectr 13: 45-53.

4. Petersen RC, Doody R, Kurz A, Mohs RC, Morris JC, et al. (2001) Current concepts in mild cognitive impairment. Arch Neurol 58: 1985-1992.

5. Petersen RC, Roberts RO, Knopman DS, Boeve BF, Geda YE, et al. (2009) Mild cognitive impairment: ten years later. Arch Neurol 66: 1447-1455.

6. Gauthier S, Scheltens $P$ (2009) Can we do better in developing new drugs for Alzheimer's disease? Alzheimers Dement 5: 489-491.

7. Albert MS, DeKosky ST, Dickson D, Dubois B, Feldman HH, et al. (2011) The diagnosis of mild cognitive impairment due to Alzheimer's disease: recommendations from the National Institute on Aging-Alzheimer's Association workgroups on diagnostic guidelines for Alzheimer's disease. Alzheimers Dement 7: 270-279.

8. Alcolea D, Martínez-Lage P, Izagirre A, Clerigué $M$, Carmona-Iragui $M$, et al. (2014) Feasibility of lumbar puncture in the study of cerebrospinal fluid 
Citation: Cintra MTG, Tavares MCS, Gomes SA, Gonçalves TdeO, Cunha LCMda, et al. (2015) P300 Evoked Potential and Risk of Mild Cognitive Impairment Progression to Alzheimer's Dementia: A Literature Review. J Neurol Neurophysiol 6: 322. doi:10.4172/2155-9562.1000322

Page 5 of 5

biomarkers for Alzheimer's disease: a multicenter study in Spain. J Alzheimers Dis 39: 719-726.

9. Campanella $S$ (2013) Why it is time to develop the use of cognitive eventrelated potentials in the treatment of psychiatric diseases. Neuropsychiatr Dis Treat 9: 1835-1845.

10. Mainardi LT, Bianchi AM, Cerutti S (1995) "Digital biomedical signal acquisition and processing" In: The Biomedical Engineering Handbook. Bronzino JD. Boca Raton: CRC Press 828-852.

11. Linden DE, Prvulovic D, Formisano E, Völlinger M, Zanella FE, et al. (1999) The functional neuroanatomy of target detection: an fMRI study of visual and auditory oddball tasks. Cereb Cortex 9: 815-823.

12. McPherson DL (1996) Long Latency auditory evoked potentials. In: Late Potentials of The auditory system. Singular Publishing Group, Inc 7-21.

13. Frizzo ACF, Alves RPC, Colafêmina JF (2001) Potenciais evocados auditivos de longa latência: um estudo comparativo entre hemisférios cerebrais. Rev Bras Otorrinolaringol 67: 618-625.

14. Polich J, Criado JR (2006) Neuropsychology and neuropharmacology of P3a and P3b. Int J Psychophysiol 60: 172-185.

15. Kraus N, McGee, T (1994) Mismatch negativity in the assessment of central auditory function. Am J Audiol 3: 39-51.

16. Gironell A, García-Sánchez C, Estévez-González A, Boltes A, Kulisevsky J (2005) Usefulness of p300 in subjective memory complaints: a prospective study. J Clin Neurophysiol 22: 279-284.

17. Squires NK, Squires KC, Hillyard SA(1975) Two varieties of long-latency positive waves evoked by unpredictable auditory stimuli in man. Electroencephalogr Clin Neurophysiol 38: 387-401.
18. Pedroso RV, Fraga FJ, Corazza DI, Andreatto CA, Coelho FG, et al. (2012) P300 latency and amplitude in Alzheimer's disease: a systematic review. Braz J Otorhinolaryngol 78: 126-132.

19. Jiang S, Qu C, Wang F, Liu Y, Qiao Z, et al. (2015) Using event-related potential $\mathrm{P} 300$ as an electrophysiological marker for differential diagnosis and to predict the progression of mild cognitive impairment: a meta-analysis. Neurol Sci 36 1105-1112.

20. Lai CL, Lin RT, Liou LM, Liu CK (2010) The role of event-related potentials in cognitive decline in Alzheimer's disease. Clin Neurophysiol 121: 194-199.

21. Papaliagkas V, Kimiskidis V, Tsolaki M, Anogianakis G (2008) Usefulness of event-related potentials in the assessment of mild cognitive impairment. BMC Neurosci 9: 107.

22. Papaliagkas VT, Anogianakis G, Tsolaki MN, Koliakos G, Kimiskidis VK (2010) Combination of P300 and CSF $\hat{l}^{2}$-amyloid (1-42) assays may provide a potential tool in the early diagnosis of Alzheimer's disease. Curr Alzheimer Res 7: 295-299.

23. Papaliagkas VT, Kimiskidis VK, Tsolaki MN, Anogianakis G (2011) Cognitive event-related potentials: longitudinal changes in mild cognitive impairment. Clin Neurophysiol 122: 1322-1326.

24. Bennys K, Rondouin G, Benattar E, Gabelle A, Touchon J (2011) Can eventrelated potential predict the progression of mild cognitive impairment? $\mathrm{J}$ Clin Neurophysiol 28: 625-632.

25. Golob EJ, Irimajiri R, Starr A (2007) Auditory cortical activity in amnestic mild cognitive impairment: relationship to subtype and conversion to dementia Brain 130: 740-752. 